\title{
On sufficient conditions for Carathéodory functions with applications
}

Adel A Attiya ${ }^{1,2^{*}}$ and Mohamed AM Nasr ${ }^{3}$

${ }^{\text {*Correspondence: }}$ aattiy@mans.edu.eg

'Department of Mathematics, Faculty of Science, University of Mansoura, Mansoura, 35516, Egypt ${ }^{2}$ Current address: Department of Mathematics, College of Science, University of Hail, Hail, Saudi Arabia Full list of author information is available at the end of the article

\section{Abstract}

In the present paper, we derive some interesting relations associated with the Carathéodory functions which yield sufficient conditions for the Carathéodory functions in the open unit disk $\mathbb{U}=\{z:|z|<1\}$. Some interesting applications of the main results are also obtained.

MSC: Primary 30C45; 30C 80

Keywords: analytic functions; starlike functions; convex functions; spirallike functions; Carathéodory functions

\section{Introduction}

Let $P$ denote the class of functions of the form

$$
p(z)=\sum_{n=0}^{\infty} p_{n} z^{n}
$$

which are analytic in the unit disc $\mathbb{U}=\{z:|z|<1\}$. The function $p(z)$ is called a Carathéodory function if it satisfies the condition

$$
\operatorname{Re}(p(z))>0 .
$$

Moreover, let $A$ denote the class of functions of the form

$$
f(z)=z+\sum_{n=2}^{\infty} a_{n} z^{n}
$$

which are analytic in the unit disc $\mathbb{U}$.

A function $f(z) \in A$ is in $K$, the class of convex functions, if it satisfies

$$
\operatorname{Re}\left(1+\frac{z f^{\prime \prime}(z)}{f(z)}\right)>0 \quad(z \in \mathbb{U})
$$

Also, a function $f(z) \in A$ is in $S^{\lambda}\left(|\lambda|<\frac{\pi}{2}\right)$, the class of $\lambda$-spirallike functions, if it satisfies

$$
\operatorname{Re}\left(e^{i \lambda} \frac{z f^{\prime}(z)}{f(z)}\right)>0 \quad(z \in \mathbb{U})
$$

Moreover, we denote by $S^{*}=S^{0}$ the class of starlike functions in $\mathbb{U}$.

\section{照 Springer}

(c) 2013 Attiya and Nasr; licensee Springer. This is an Open Access article distributed under the terms of the Creative Commons Attribution License (http://creativecommons.org/licenses/by/2.0), which permits unrestricted use, distribution, and reproduction in any medium, provided the original work is properly cited. 
Definition 1.1 Let $f(z)$ and $F(z)$ be analytic functions. The function $f(z)$ is said to be $s u b$ ordinate to $F(z)$, written $f(z) \prec F(z)$, if there exists a function $w(z)$ analytic in $\mathbb{U}$, with $w(0)=0$ and $|w(z)| \leq 1$, and such that $f(z)=F(w(z))$. If $F(z)$ is univalent, then $f(z) \prec F(z)$ if and only if $f(0)=F(0)$ and $f(\mathbb{U}) \subset F(\mathbb{U})$.

Definition 1.2 Let $\mathbb{D}$ be the set of analytic functions $q(z)$ and injective on $\overline{\mathbb{U}} \backslash E(q)$, where

$$
E(q)=\left\{\zeta \in \partial \mathbb{U}: \lim _{z \rightarrow \zeta} q(z)=\infty\right\}
$$

and $q^{\prime}(\zeta) \neq 0$ for $\zeta \in \partial \mathbb{U} \backslash E(q)$. Further, let $\mathbb{D}_{a}=\{q(z) \in \mathbb{D}: q(0)=a\}$.

Many authors have obtained several relations of Carathéodory functions, e.g., see $([1-13])$.

In the present paper, we derive some relations associated with the Carathéodory functions which yield the sufficient conditions for Carathéodory functions in $\mathbb{U}$. Some applications of the main results are also obtained.

\section{Main results}

To prove our results, we need the following lemma due to Miller and Mocanu [14, p.24]

Lemma 2.1 Let $q(z) \in \mathbb{D}_{a}$ and let

$$
p(z)=b+b_{n} z^{n}+\cdots
$$

be analytic in $\mathbb{U}$ with $p(z) \neq b$. If $p(z) \nprec q(z)$, then there exist points $z_{0} \in \mathbb{U}$ and $\zeta_{0} \in \partial \mathbb{U} \backslash E(q)$ and on $m \geq n \geq 1$ for which

(i) $p\left(z_{0}\right)=q\left(\zeta_{0}\right)$,

(ii) $z_{0} p^{\prime}\left(z_{0}\right)=m \zeta_{0} q^{\prime}\left(\zeta_{0}\right)$.

Theorem 2.1 Let

$$
P: \mathbb{U} \rightarrow \mathbb{C}
$$

with

$$
\operatorname{Re}(\bar{a} P(z))>0 \quad(a \in \mathbb{C}) .
$$

If $p(z)$ is an analytic function in $\mathbb{U}$ with $p(0)=1$ and

$$
\operatorname{Re}\left(p(z)+P(z) z p^{\prime}(z)\right)>\frac{E}{2|a|^{2} \operatorname{Re}(\bar{a} P(z))},
$$

then

$$
\operatorname{Re}(a p(z))>0,
$$

where

$$
E=-(\operatorname{Re}(a))(\operatorname{Re}(\bar{a} P(z)))^{2}+2 \operatorname{Re}(\bar{a} P(z))(\operatorname{Im}(a))^{2}+(\operatorname{Re}(a))(\operatorname{Im}(a))^{2}
$$

with $\operatorname{Re}(a)>0$. 
Proof Let us define both $q(z)$ and $h(z)$ as follows:

$$
q(z)=\operatorname{ap}(z)
$$

and

$$
h(z)=\frac{a+\bar{a} z}{1-z} \quad(\operatorname{Re}(a)>0),
$$

where $p(z)$ is defined by (2.1) since $q(z)$ and $h(z)$ are analytic functions in $\mathbb{U}$ with $q(0)=$ $h(0)=a \in \mathbb{C}$ with

$$
h(\mathbb{U})=\{w: \operatorname{Re}(w)>0\} .
$$

Now, we suppose that $q(z) \nprec h(z)$. Therefore, by using Lemma 2.1, there exist points

$$
z_{0} \in \mathbb{U} \quad \text { and } \quad \zeta_{0} \in \partial \mathbb{U} \backslash\{1\}
$$

such that $q\left(z_{0}\right)=h\left(\zeta_{0}\right)$ and $z_{0} q^{\prime}\left(z_{0}\right)=m \zeta_{0} h^{\prime}\left(\zeta_{0}\right), m \geq n \geq 1$.

We note that

$$
\zeta_{0}=h^{-1}\left(q\left(z_{0}\right)\right)=\frac{q\left(z_{0}\right)-a}{q\left(z_{0}\right)+\bar{a}}
$$

and

$$
\varsigma_{0} h^{\prime}\left(\varsigma_{0}\right)=-\frac{\left|q\left(z_{0}\right)-a\right|^{2}}{2 \operatorname{Re}\left(a-q\left(z_{0}\right)\right)} .
$$

We have $h\left(\zeta_{0}\right)=\rho i(\rho \in \mathbb{R})$; therefore,

$$
\begin{aligned}
\operatorname{Re} & \left(p\left(z_{0}\right)+P\left(z_{0}\right) z p^{\prime}\left(z_{0}\right)\right) \\
& =\operatorname{Re}\left(\frac{1}{a} h\left(\zeta_{0}\right)+\frac{1}{a} P\left(z_{0}\right) m \zeta_{0} h^{\prime}\left(\zeta_{0}\right)\right) \\
& =\operatorname{Re}\left(\frac{\rho i}{a}\right)-m \frac{|\rho i-a|^{2}}{2 \operatorname{Re}(a)} \operatorname{Re}\left(\frac{P\left(z_{0}\right)}{a}\right) \\
& \leq \operatorname{Re}\left(\frac{\rho i}{a}\right)-\frac{|\rho i-a|^{2}}{2 \operatorname{Re}(a)} \operatorname{Re}\left(\frac{P\left(z_{0}\right)}{a}\right) \\
& =A \rho^{2}+B \rho+C \\
& =g(\rho),
\end{aligned}
$$

where

$$
\begin{aligned}
& A=-\frac{\operatorname{Re}\left(\bar{a} p\left(z_{0}\right)\right)}{2|a|^{2} \operatorname{Re}(a)}, \\
& B=\frac{\operatorname{Im}(a)}{|a|^{2}}\left(1+\frac{\operatorname{Re}\left(\bar{a} p\left(z_{0}\right)\right)}{\operatorname{Re}(a)}\right)
\end{aligned}
$$


and

$$
C=-\frac{\operatorname{Re}\left(\bar{a} p\left(z_{0}\right)\right)}{2 \operatorname{Re}(a)} .
$$

We can see that the function $g(\rho)$ in (2.6) takes the maximum value at $\rho_{1}$ given by

$$
\rho_{1}=\operatorname{Im}(a)\left(1+\frac{\operatorname{Re}(a)}{\operatorname{Re}\left(\bar{a} p\left(z_{0}\right)\right)}\right)
$$

Hence, we have

$$
\begin{aligned}
\operatorname{Re}\left(p\left(z_{0}\right)+P\left(z_{0}\right) z p^{\prime}\left(z_{0}\right)\right) & \leq g\left(\rho_{1}\right) \\
& =\frac{E}{2|a|^{2} \operatorname{Re}(\bar{a} P(z))},
\end{aligned}
$$

where $E$ is defined by (2.3). This is a contradiction to (2.2). Then we obtain $\operatorname{Re}(a p(z))>0$.

Theorem 2.2 Let $p(z)$ be a nonzero analytic function in $\mathbb{U}$ and $p(0)=1$. If

$$
\gamma_{1}<\operatorname{Im}\left(p(z)+\frac{z p^{\prime}(z)}{p(z)}\right)<\gamma_{2},
$$

where

$$
\gamma_{1}=-\frac{\sqrt{|a|^{2}+2(\operatorname{Re}(a))^{2}}-\operatorname{Im}(a)}{\operatorname{Re} a}
$$

and

$$
\gamma_{2}=\frac{\sqrt{|a|^{2}+2(\operatorname{Re}(a))^{2}}+\operatorname{Im}(a)}{\operatorname{Re}(a)},
$$

then

$$
\operatorname{Re}(\operatorname{ap}(z))>0
$$

where $\operatorname{Re}(a)>0$.

Proof Let us define both $q(z)$ and $h(z)$ as follows:

$$
q(z)=\operatorname{ap}(z)
$$

and

$$
h(z)=\frac{a+\bar{a} z}{1-z} \quad(\operatorname{Re}(a)>0)
$$

where $p(z)$ is defined by (2.1) since $q(z)$ and $h(z)$ are analytic functions in $\mathbb{U}$ with $q(0)=$ $h(0)=a \in \mathbb{C}$ with

$$
h(\mathbb{U})=\{w: \operatorname{Re}(w)>0\} .
$$


Now, we suppose that $q(z) \nprec h(z)$. Therefore, by using Lemma 2.1, there exist points

$$
z_{0} \in \mathbb{U} \quad \text { and } \quad \zeta_{0} \in \partial \mathbb{U} \backslash\{1\}
$$

such that $q\left(z_{0}\right)=h\left(\zeta_{0}\right)$ and $z_{0} q^{\prime}\left(z_{0}\right)=m \zeta_{0} h^{\prime}\left(\zeta_{0}\right), m \geq n \geq 1$.

We note that

$$
\zeta_{0} h^{\prime}\left(\zeta_{0}\right)=-\frac{\left|q\left(z_{0}\right)-a\right|^{2}}{2 \operatorname{Re}\left(a-q\left(z_{0}\right)\right)}
$$

We have $h\left(\zeta_{0}\right)=\rho i(\rho \in \mathbb{R})$; therefore,

$$
\begin{aligned}
\operatorname{Im}\left(p\left(z_{0}\right)+\frac{z_{0} p^{\prime}\left(z_{0}\right)}{p\left(z_{0}\right)}\right) & =\operatorname{Im}\left(q\left(z_{0}\right)+\frac{z_{0} q^{\prime}\left(z_{0}\right)}{q\left(z_{0}\right)}\right) \\
& =\operatorname{Im}\left(\frac{h\left(\zeta_{0}\right)}{a}+\frac{m \zeta_{0} h^{\prime}\left(\zeta_{0}\right)}{h\left(\zeta_{0}\right)}\right) \\
& =\operatorname{Im}\left(\frac{\rho i}{a}-\frac{m|\rho i-a|^{2}}{2 \operatorname{Re}(a) \rho i}\right) \\
& =\frac{\rho}{|a|^{2}} \operatorname{Re}(a)+\frac{m|\rho i-a|^{2}}{2 \rho \operatorname{Re}(a)}
\end{aligned}
$$

For the case $\rho>0$, we obtain

$$
\begin{aligned}
\operatorname{Im}\left(p\left(z_{0}\right)+\frac{z_{0} p^{\prime}\left(z_{0}\right)}{p\left(z_{0}\right)}\right) & \geq \frac{\rho}{|a|^{2}} \operatorname{Re}(a)+\frac{|\rho i-a|^{2}}{2 \rho \operatorname{Re}(a)} \\
& =\frac{1}{2 \rho \operatorname{Re}(a)}\left[\left(1+2\left(\frac{\operatorname{Re}(a)}{|a|}\right)^{2}\right) \rho^{2}+2 \operatorname{Im}(a) \rho+|a|\right] \\
& =g(\rho) .
\end{aligned}
$$

We can see that the function $g(\rho)$ in (2.9) takes the minimum value at $\rho_{1}$ given by

$$
\rho_{1}=\frac{|a|^{2}}{\sqrt{|a|^{2}+2(\operatorname{Re}(a))^{2}}} .
$$

Hence, we have

$$
\begin{aligned}
\operatorname{Im}\left(p\left(z_{0}\right)+\frac{z_{0} p^{\prime}\left(z_{0}\right)}{p\left(z_{0}\right)}\right) & \geq g\left(\rho_{1}\right) \\
& =\gamma_{2} .
\end{aligned}
$$

This is a contradiction to (2.7). Then we obtain $\operatorname{Re}(a p(z))>0$.

For the case $\rho<0$, we obtain

$$
\begin{aligned}
\operatorname{Im}\left(p\left(z_{0}\right)+\frac{z_{0} p^{\prime}\left(z_{0}\right)}{p\left(z_{0}\right)}\right) & \leq \frac{\rho}{|a|^{2}} \operatorname{Re}(a)+\frac{|\rho i-a|^{2}}{2 \rho \operatorname{Re}(a)} \\
& =\frac{1}{2 \rho \operatorname{Re}(a)}\left[\left(1+2\left(\frac{\operatorname{Re}(a)}{|a|}\right)^{2}\right) \rho^{2}+2 \operatorname{Im}(a) \rho+|a|^{2}\right] \\
& =g(\rho) .
\end{aligned}
$$


We can see that the function $g(\rho)$ in (2.10) takes the maximum value at $\rho_{2}$ given by

$$
\rho_{2}=-\frac{|a|^{2}}{\sqrt{|a|^{2}+2(\operatorname{Re}(a))^{2}}} .
$$

Hence, we have

$$
\begin{aligned}
\operatorname{Im}\left(p\left(z_{0}\right)+\frac{z_{0} p^{\prime}\left(z_{0}\right)}{p\left(z_{0}\right)}\right) & \leq g\left(\rho_{2}\right) \\
& =\gamma_{1} .
\end{aligned}
$$

This is a contradiction to (2.7). Then we obtain $\operatorname{Re}(a p(z))>0$.

Theorem 2.3 Let $p(z)$ be a nonzero analytic function in $\mathbb{U}$ with $p(0)=1$. If

$$
\left|p(z)+\frac{z p^{\prime}(z)}{p(z)}-1\right|<\frac{3 \operatorname{Re}(a)}{2|a|}
$$

then

$$
\operatorname{Re}\left(\frac{a}{p(z)}\right)>0
$$

where $\operatorname{Re}(a)>0$.

Proof Let us define both $q(z)$ and $h(z)$ as follows:

$$
q(z)=\operatorname{ap}(z)
$$

and

$$
h(z)=\frac{a+\bar{a} z}{1-z} \quad(\operatorname{Re}(a)>0),
$$

where $p(z)$ is defined by (2.1) since $q(z)$ and $h(z)$ are analytic functions in $\mathbb{U}$ with $q(0)=$ $h(0)=a \in \mathbb{C}$ with

$$
h(\mathbb{U})=\{w: \operatorname{Re} w>0\} .
$$

Now, we suppose that $q(z) \nprec h(z)$. Therefore, by using Lemma 2.1, there exist points

$$
z_{0} \in \mathbb{U} \quad \text { and } \quad \zeta_{0} \in \partial \mathbb{U} \backslash\{1\}
$$

such that $q\left(z_{0}\right)=h\left(\zeta_{0}\right)$ and $z_{0} q^{\prime}\left(z_{0}\right)=m \zeta_{0} h^{\prime}\left(\zeta_{0}\right), m \geq n \geq 1$.

We note that

$$
\zeta_{0} h^{\prime}\left(\zeta_{0}\right)=-\frac{\left|q\left(z_{0}\right)-a\right|^{2}}{2 \operatorname{Re}\left(a-q\left(z_{0}\right)\right)}
$$

We have $h\left(\zeta_{0}\right)=\rho i(\rho \in \mathbb{R})$. 
Therefore,

$$
\begin{aligned}
\frac{\left|p\left(z_{0}\right)+\frac{z p^{\prime}\left(z_{0}\right)}{p\left(z_{0}\right)}-1\right|}{\left|p\left(z_{0}\right)\right|} & =\left|\frac{\rho i}{a}-\frac{m}{a} \frac{|a-i \rho|^{2}}{2 \operatorname{Re}(a)}-1\right| \\
& \geq \frac{1}{|a|}\left|\frac{m|a-i \rho|^{2}}{2 \operatorname{Re}(a)}+\operatorname{Re}(a)\right| \\
& \geq \frac{1}{|a|}\left(\frac{|a-i \rho|^{2}}{2 \operatorname{Re}(a)}+\operatorname{Re}(a)\right) \\
& \geq \frac{1}{2|a| \operatorname{Re}(a)}\left(3(\operatorname{Re}(a))^{2}+(\operatorname{Im}(a)-\rho)^{2}\right) \\
& \geq \frac{3 \operatorname{Re}(a)}{2|a|} .
\end{aligned}
$$

This is a contradiction to (2.7). Then we obtain $\operatorname{Re}\left(\frac{a}{p(z)}\right)>0$.

\section{Applications and examples}

Putting $P(z)=\beta(\beta>0$; real $)$ in Theorem 2.1 , we have the following corollary.

Corollary 3.1 If $p(z)$ is an analytic function in $\mathbb{U}$ with $p(0)=1$ and

$$
\operatorname{Re}\left(p(z)+\beta z p^{\prime}(z)\right)>\frac{E}{2 \beta|a|^{2} \operatorname{Re}(a)},
$$

then

$$
\operatorname{Re}(\operatorname{ap}(z))>0
$$

where

$$
E=-(\operatorname{Re}(a))\left[\beta^{2}(\operatorname{Re}(a))^{2}+(1+2 \beta)(\operatorname{Im}(a))^{2}\right]
$$

with $\operatorname{Re}(a)>0$.

Putting $\beta=1$ in Corollary 3.1, we obtain the following corollary.

Corollary 3.2 If $p(z)$ is an analytic function in $\mathbb{U}$ with $p(0)=1$ and

$$
\operatorname{Re}\left(p(z)+z p^{\prime}(z)\right)>\frac{3}{2}-2\left(\frac{\operatorname{Re}(a)}{|a|}\right)^{2}
$$

then

$$
\operatorname{Re}(a p(z))>0
$$

where $\operatorname{Re}(a)>0$.

Putting $p(z)=\frac{f(z)}{g(z)}$ and $P(z)=\frac{g(z)}{z g^{\prime}(z)}$ in Theorem 2.1, we have the following corollary. 
Corollary 3.3 Let $f(z) \in A, g(z) \in S^{*}$ and

$$
\operatorname{Re}\left(\frac{f^{\prime}(z)}{g^{\prime}(z)}\right)>\frac{3}{2}-2\left(\frac{\operatorname{Re}(a)}{|a|}\right)^{2}
$$

Then

$$
\operatorname{Re}\left(a \frac{f(z)}{g(z)}\right)>0
$$

where $\operatorname{Re}(a)>0$.

Example 3.1 Let $f(z) \in A$ satisfy the following relation:

$$
\operatorname{Re}\left(f^{\prime}(z)\right)>\frac{3}{2}-2\left(\frac{\operatorname{Re}(a)}{|a|}\right)^{2} .
$$

Then

$$
\operatorname{Re}\left(a \frac{f(z)}{z}\right)>0
$$

where $\operatorname{Re}(a)>0$.

Example 3.2 Let $f(z) \in A$ satisfy the following relation:

$$
\operatorname{Re}\left(\left(2+\frac{z f^{\prime \prime}(z)}{f^{\prime}(z)}-\frac{z f^{\prime}(z)}{f(z)}\right) \frac{z f^{\prime}(z)}{f(z)}\right)>\frac{3}{2}-2\left(\frac{\operatorname{Re}(a)}{|a|}\right)^{2} .
$$

Then

$$
\operatorname{Re}\left(a \frac{z f^{\prime}(z)}{f(z)}\right)>0
$$

where $\operatorname{Re}(a)>0$.

\section{Remark 3.1}

(i) Putting $a=e^{i \lambda}\left(|\lambda|<\frac{\pi}{2}\right)$ in Theorem 2.1, we have Theorem 1 due to Kim and Cho [3].

(ii) Putting $a=e^{i \lambda}\left(|\lambda|<\frac{\pi}{2}\right), P(z)=\beta(\beta>0$; real) in Theorem 2.1, we have Corollary 1 due to Kim and Cho [3].

(iii) Putting $a=0$ and $P(z)=1$ in Theorem 2.1, we have the result due to Nunokawa $e t$ al. [15].

(iv) Putting $a=e^{i \lambda}\left(|\lambda|<\frac{\pi}{2}\right), P(z)=1$ in Theorem 2.1, we have Corollary 2 due to Kim and Cho [3].

Putting $p(z)=\frac{z f^{\prime}(z)}{f(z)}$ in Theorem 2.2, we have the following corollary.

Corollary 3.4 Let $f(z) \in A$. If

$$
\gamma_{1}<\operatorname{Im}\left(1+\frac{z f^{\prime \prime}(z)}{f^{\prime}(z)}\right)<\gamma_{2}
$$


where

$$
\gamma_{1}=-\frac{\sqrt{|a|^{2}+2(\operatorname{Re}(a))^{2}}-\operatorname{Im}(a)}{\operatorname{Re}(a)}
$$

and

$$
\gamma_{2}=\frac{\sqrt{|a|^{2}+2(\operatorname{Re}(a))^{2}}+\operatorname{Im}(a)}{\operatorname{Re}(a)},
$$

then

$$
\operatorname{Re}\left(a \frac{z f^{\prime}(z)}{f(z)}\right)>0
$$

where $\operatorname{Re}(a)>0$.

Putting $p(z)=\frac{z f^{\prime}(z)}{f(z)}$ in Theorem 2.3, we have the following corollary.

Corollary 3.5 Let $p(z)$ be a nonzero analytic function in $\mathbb{U}$ with $p(0)=1$. If

$$
\left|\frac{z f^{\prime \prime}(z)}{f^{\prime}(z)}\right|<\frac{3 \operatorname{Re}(a)}{2|a|}
$$

then

$$
\operatorname{Re}\left(\frac{1}{a} \frac{z f^{\prime}(z)}{f(z)}\right)>0
$$

where $\operatorname{Re}(a)>0$.

Remark 3.2 Putting $a=e^{i \lambda}\left(|\lambda|<\frac{\pi}{2}\right)$ in Corollary 3.5, we have the result due to Kim and Cho [3].

\section{Competing interests}

The authors declare that they have no competing interests.

\section{Authors' contributions}

All authors contributed equally to the paper. Also, all authors have read and approved the final version of the paper.

\section{Author details}

${ }^{1}$ Department of Mathematics, Faculty of Science, University of Mansoura, Mansoura, 35516, Egypt. ${ }^{2}$ Current address: Department of Mathematics, College of Science, University of Hail, Hail, Saudi Arabia. ${ }^{3}$ Department of Mathematics, College of Science, King Khaled University, Abha, Saudi Arabia.

\section{Acknowledgements}

Dedicated to Professor Hari M Srivastava.

The authors would like to express their gratitude to the referees for the valuable advices to improve this paper.

Received: 25 November 2012 Accepted: 16 January 2013 Published: 19 April 2013

\section{References}

1. Cho, NE, Hwa, Kl: Conditions for Carathéodory functions. J. Inequal. Appl. 2009, Article ID 601597 (2009)

2. Delsarte, P, Genin, Y: A simple proof of Livingston's inequality for Carathéodory functions. Proc. Am. Math. Soc. 107, 1017-1020 (1989)

3. Kim, IH, Cho, NE: Sufficient conditions for Carathéodory functions. Comput. Math. Appl. 59(6), 2067-2073 (2010)

4. Li, JL, Owa, S: Sufficient conditions for starlikeness. Indian J. Pure Appl. Math. 33, 313-318 (2002) 
5. Miller, S: Differential inequalities and Carathéodory functions. Bull. Am. Math. Soc. 81, 79-81 (1975)

6. Nunokawa, M: Differential inequalities and Carathéodory functions. Proc. Jpn. Acad., Ser. A, Math. Sci. 65, 326-328 (1989)

7. Nunokawa, M: On properties of non-Carathéodory functions. Proc. Jpn. Acad., Ser. A, Math. Sci. 68, 152-153 (1992)

8. Nunokawa, M, Owa, S, Takahashi, N, Saitoh, H: Sufficient conditions for Carathéodory functions. Indian J. Pure Appl. Math. 33, 1385-1390 (2002)

9. Obradović, M, Owa, S: On certain properties for some classes of starlike functions. J. Math. Anal. Appl. 145, 357-364 (1990)

10. Padmanabhan, KS: On sufficient conditions for starlikeness. Indian J. Pure Appl. Math. 32, 543-550 (1990)

11. Shiraishi, H, Owa, S, Srivastava, HM: Sufficient conditions for strongly Carathéodory functions. Comput. Math. Appl. 62(8), 2978-2987 (2011)

12. Tuneski, N: On certain sufficient conditions for starlikeness. Int. J. Math. Math. Sci. 23, 521-527 (2000)

13. Yang, D, Owa, S, Ochiai, K: Sufficient conditions for Carathéodory functions. Comput. Math. Appl. 51, 467-474 (2006)

14. Miller, SS, Mocanu, PT: Differential Subordination, Theory and Application. Dekker, New York (2000)

15. Nunokawa, M, Owa, S, Nishiwaki, J, Saitoh, H: Sufficient conditions for starlikeness and convexity of analytic functions with real coefficients. Southeast Asian Bull. Math. 33(6), 1149-1155 (2009)

doi:10.1186/1029-242X-2013-191

Cite this article as: Attiya and Nasr: On sufficient conditions for Carathéodory functions with applications. Journal of Inequalities and Applications 2013 2013:191.

\section{Submit your manuscript to a SpringerOpen ${ }^{\circ}$ journal and benefit from:}

- Convenient online submission

Rigorous peer review

- Immediate publication on acceptance

- Open access: articles freely available online

- High visibility within the field

- Retaining the copyright to your article 